\title{
Improved Variant Calling Accuracy by Merging Replicates in Whole-Exome Sequencing Studies
}

\author{
Yanfeng Zhang, ${ }^{1}$ Bingshan Li, ${ }^{2}$ Chun Li, ${ }^{3}$ Qiuyin Cai, ${ }^{1}$ Wei Zheng, ${ }^{1}$ and Jirong Long ${ }^{1}$ \\ ${ }^{1}$ Division of Epidemiology, Department of Medicine, Vanderbilt Epidemiology Center, Vanderbilt-Ingram Cancer Center, \\ Vanderbilt University School of Medicine, Nashville, TN 37203, USA \\ ${ }^{2}$ Department of Molecular Physiology and Biophysics, Center for Human Genetics Research, Vanderbilt University School of Medicine, \\ Nashville, TN 37232, USA \\ ${ }^{3}$ Department of Biostatistics, Vanderbilt University School of Medicine, Nashville, TN 37232, USA
}

Correspondence should be addressed to Jirong Long; jirong.long@vanderbilt.edu

Received 28 May 2014; Revised 15 July 2014; Accepted 15 July 2014; Published 4 August 2014

Academic Editor: Ernesto Picardi

Copyright (C) 2014 Yanfeng Zhang et al. This is an open access article distributed under the Creative Commons Attribution License, which permits unrestricted use, distribution, and reproduction in any medium, provided the original work is properly cited.

In large scale population-based whole-exome sequencing (WES) studies, there are some samples occasionally sequenced two or more times due to a variety of reasons. To investigate how to efficiently utilize these duplicated sequencing data, we conducted comprehensive evaluation of variant calling strategies. 92 samples subjected to WES twice were selected from a large population study. These 92 duplicated samples were divided into two groups: group $\mathrm{H}$ consisting of the higher sequencing depth for each subject and group L consisting of the lower depth for each subject. The merged samples for each subject were put in a third group M. Using the GATK multisample toolkit, we compared variant calling accuracy among three strategies. Hierarchical clustering analysis indicated that the two replicates for each subject showed high homogeneity. The comparative analyses on the basis of heterozygous-homozygous ratio (Hete/Homo), transition-transversion ratio (Ti/Tv), and overlapping rate with the $1000 \mathrm{Genomes}$ Project consistently showed that the data quality of the SNPs detected from the M group was more accurate than that of SNPs detected from the $\mathrm{H}$ and $\mathrm{L}$ groups. These results suggested that merging homogeneous duplicated exomes instead of using one of them could improve variant calling accuracy.

\section{Introduction}

Next generation sequencing technologies generate huge amount of data in a single experimental run and provide a revolutionary tool for various genomics studies [1]. Primarily designed to capture the intended coding variants [2-4], whole-exome sequencing (WES) has been commonly used in basic and translational research, including investigation of diversity and demographic history in human populations [5-8], identification of etiological variants [9-13], crossspecies genome comparison $[14,15]$, and even phylogenetic estimation [16].

Among currently available exome enrichment platforms, some platforms such as Illumina TruSeq were also designed to capture a portion of noncoding regions, including untranslated regions (UTRs) and intronic regions [17]. In addition, we previously found that a significant amount of DNA fragments from WES falls outside the intended regions and a large portion of these fragments is of high quality [18].
In large WES studies, some samples are occasionally sequenced twice or even more times due to a variety of reasons, for example, insufficient coverage in the first experiment, sample duplication, and the rest. It is challenging how to best utilize these duplicated exomes for SNP discovery and genotype calling, especially with batch effects taken into consideration. In the present study, we systematically evaluated SNP detection performance of three strategies to utilize the duplicated exome data of 92 subjects, only using the data with higher depth, only using the data with lower depth, and using the merged data from technical replicates.

\section{Materials and Methods}

2.1. Sample Collection. The subjects in this study were a subset of participants in the Shanghai Breast Cancer Study (SBCS), which was a population-based breast cancer case control study. Details of the study have been described elsewhere 
$[19,20]$. In brief, the SBCS is a population-based, case control study including 3,448 breast cancer cases and 3,474 controls. Of these, 92 subjects (51 cases and 41 controls) were included in the current investigation. Genomic DNA from buffy coat was extracted using QIAmp DNA kit (Qiagen, Valencia, CA) following the manufacture's protocol. Approval of the study was granted by the relevant institutional review boards in both China and the United States.

2.2. Exome Sequencing and Variant Calling. For each of 92 subjects, one sequencing library was constructed for genomic DNA captured on the Illumina TruSeq platform according to the manufacturer's instructions and subjected to 75 or $100 \mathrm{bp}$ paired end sequencing twice on the Illumina HiSeq instrument. For each exome, raw reads in FASTQ format were aligned to the human reference genome (hg19) using Burrows-Wheeler Aligner (BWA, v0.5.9) in default parameters [21]. Mapped data were then processed and sorted using SAMtools [22]. Local realignment, PCR duplicates removal, and base quality score recalibration (BQSR) were performed using Genome Analysis toolkit (GATK) to generate BAM files [23]. A total of 184 BAM files were then divided into two groups, high depth $(\mathrm{H})$ and low depth (L) from each subject, based on the mean depth across on-target regions. We also merged the two BAM files for each subject into a single BAM file and combined 92 merged BAM files as a third group $(\mathrm{M})$. We called SNPs within each group $(\mathrm{H}, \mathrm{L}$, and $\mathrm{M}$ ) via the GATK multisample strategy [24] (Figure 1). Then, we conducted the variants filtering as follows: (1) $\geq$ 3 SNPs detected within 10 bp distance; (2) > 10\% alignments mapped ambiguously; (3) SNPs having a quality score $<50$; (4) variant confidence/quality by depth $<1.5$; (5) strand bias score calculated by GATK > -1 .

2.3. Statistics of Exome-Sequencing Performance. The metric of RPKM (reads per kilobase and million mapped reads) is a commonly used method for RNA-sequencing data normalization. Recently, the RPKM was also used to evaluate the performance of exome sequencing [25]. In this study, the RPKM was employed and calculated as the number of reads aligned on the target region per kilobase of target sequence divided by the total number of mapped reads. The reads aligned to the $100 \mathrm{bp}$ upstream or downstream of the target regions were also included in the PRKM statistic [25]. The Euclidean distance among the 184 exomes was calculated based on the RPKM value. We conducted the unsupervised hierarchical clustering analysis using $\mathrm{R}$ programming language (version 2.15.1) to evaluate batch effects among 92 replicated sequencing experiments.

2.4. SNP Detection Performance. We compared SNP detection performance for each of three SNP calling strategies from the following aspects: (1) the total number of SNPs observed; (2) the ratio of heterozygous genotypes to nonreference homozygous genotypes (Hete/Homo), which is expected to be close to 1.5 on genomic scale $[26,27]$; (3) the transitiontransversion ratio $(\mathrm{Ti} / \mathrm{Tv})$, which is expected to be $\sim 2$ across the whole genome and $\sim 3$ across the exon regions $[18,27]$;

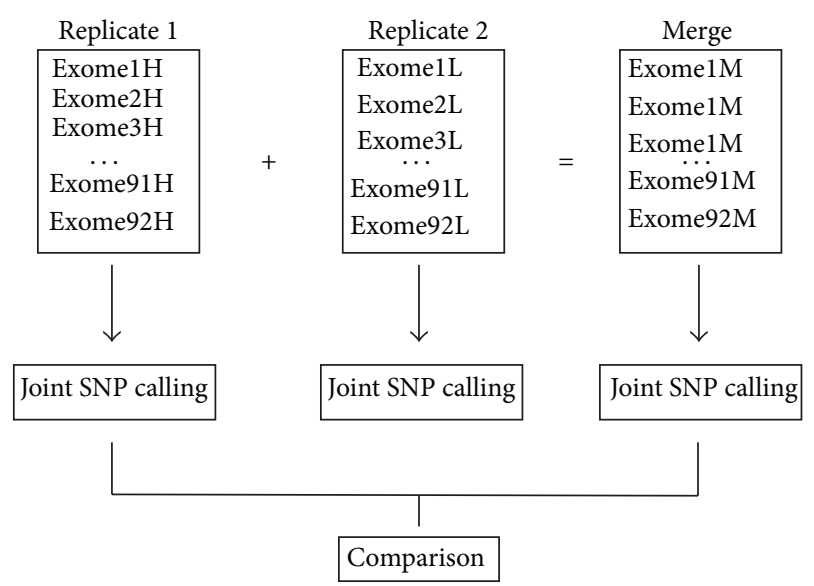

FIGURE 1: Schematic of SNP calling strategies in this study. For 92 subjects with duplicates, we divided them into two groups (replicate 1 and replicate 2) according to mean sequencing depth across the target regions. The replicate 1 group is comprised of exomes with the relative high mean depth (marked with ExomelH to Exome92H). The remainders with the relative low depth are grouped as replicate 2. Merging BAM files from the two duplicates generates a third group, referred to as the merged group flagged with ExomelM to Exome92M.

(4) the overlapping rate between the SNPs uniquely detected within each group with the SNPs observed in the 1000 Genomes (1 KG) Project [28]. If a closer approximation to the empirically expected value as well as a higher overlapping rate is observed, it generally manifests lower false discovery rate. The evaluation was inherently stratified, according to the locations of SNPs, to on-target regions and off-target regions. All analyses were achieved by a series of custom Perl scripts.

\section{Results}

3.1. Data Generation between the Duplicates. Table 1 summarizes the statistics for the exome sequencing data. We obtained an average of 64.0 and 57.2 million reads per exome, with 43.4 and 36.0 mean depths across the target regions, for the $\mathrm{H}$ and $\mathrm{L}$ group, respectively (see Supplementary Table 1 in Supplementary Material available online at http://dx.doi.org/10.1155/2014/319534). On average, $98.23 \%$ and $98.65 \%$ of the reads were aligned to the human reference genome, and $49.70 \%$ and $49.11 \%$ were mapped to the target regions, in the $\mathrm{H}$ and $\mathrm{L}$ groups, respectively. Approximately $86.16 \%$ and $86.14 \%$ of the reads in the $\mathrm{H}$ and $\mathrm{L}$ groups had mapping quality $\geq 20$, respectively. Overall, the comparative results showed the high homogeneity between the duplicates for each subject (Supplementary Table 1). Furthermore, the hierarchical clustering result based on PRKM values also showed that the two replicates for each subject were closely clustered with each other (Figure 2). These results suggest there is low heterogeneity between the replicated experiments.

3.2. SNP Detection Performance. First, we compared the number of SNPs called from different strategies. Figure 3 summarizes the number of SNPs detected in each exome. For 


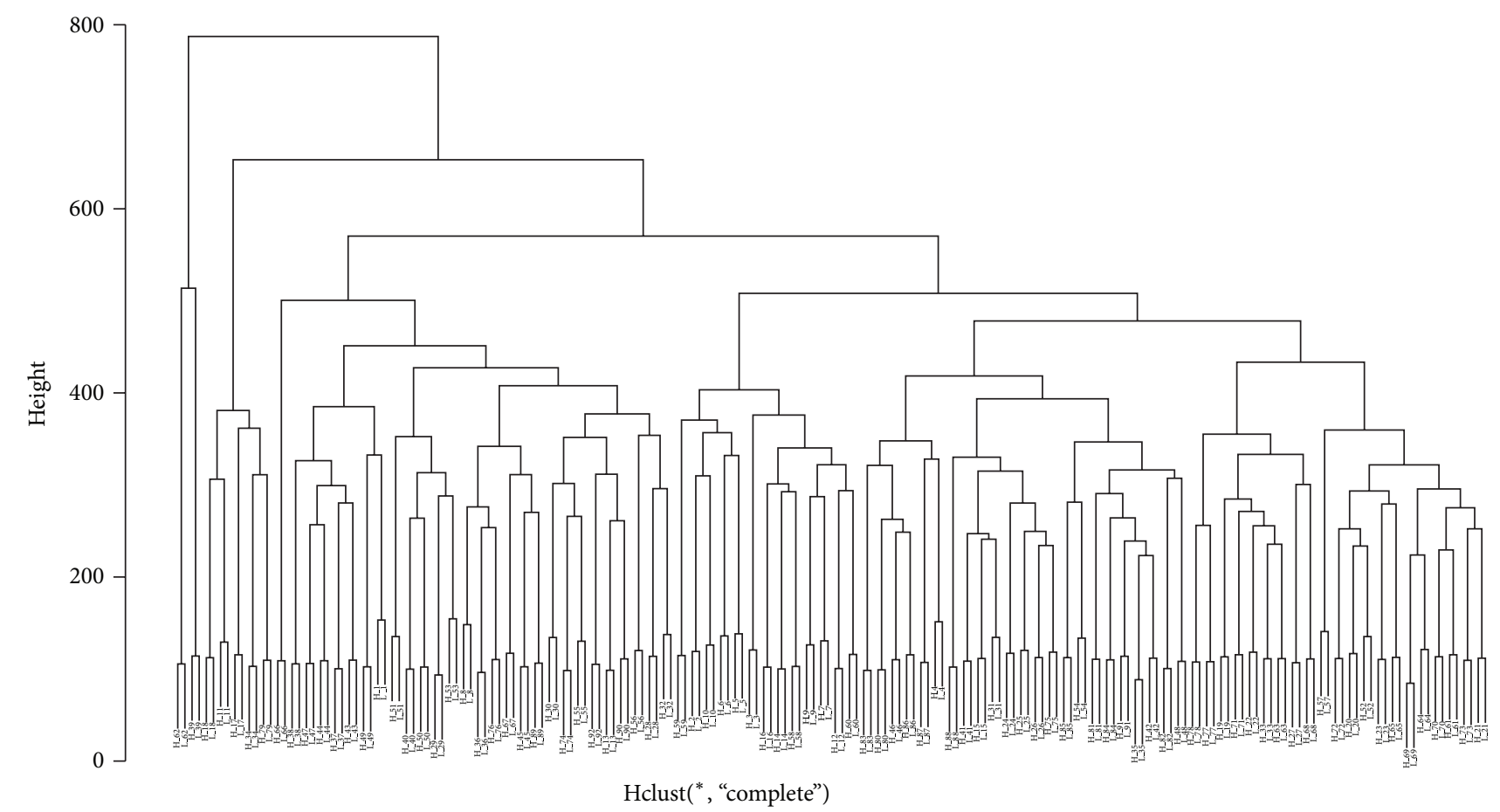

FIGURE 2: Hierarchical clustering of replicated exomes of 92 subjects. The pairwise distance is based on the RPKM matrix for each exome. Duplicates within each subject are flagged with prefix of $\mathrm{H}$ or $\mathrm{L}$.

TABLE 1: Summary of WES samples divided into two groups ( $\mathrm{H}$ and $\mathrm{L})$.

\begin{tabular}{lcccccc}
\hline Group & Total reads $\left(\times 10^{6}\right)$ & Mapping rate (\%) & Target mapping (\%) & Mean depth ${ }^{\mathrm{a}}$ & $\begin{array}{c}\text { Mapping rate with } \\
\text { MQ }<10(\%)^{\mathrm{b}}\end{array}$ & $\begin{array}{c}\text { Mapping rate with } \\
\text { MQ } \geq 20(\%)^{\mathrm{b}}\end{array}$ \\
\hline $\mathrm{H}$ & $64.0(49.2-97.9)$ & $98.2(95.9-99.2)$ & $49.7(46.6-55.4)$ & $43.4(34.2-65.8)$ & $10.4(9.5-11.3)$ & $86.2(84.0-87.7)$ \\
$\mathrm{L}$ & $57.2(40.3-79.3)$ & $98.7(97.6-99.2)$ & $49.1(44.5-55.0)$ & $36.0(29.5-53.0)$ & $10.8(9.6-12.3)$ & $86.1(84.4-87.6)$ \\
\hline
\end{tabular}

${ }^{\mathrm{a}}$ Mean depth across target regions.

${ }^{\mathrm{b}} \mathrm{MQ}$ denotes mapping quality.

Note that the range of values is shown in the parenthesis.

the target regions, the average number of SNPs observed in each exome was similar across the three groups, with 46,860 , 44,806 , and 43,664 for the M, H, and L groups, respectively (Figure 3(a)). Among these SNPs across the TruSeq target regions detected in the $\mathrm{M}$ group, $95.0 \%$ and $92.5 \%$ of them were also observed in the $\mathrm{H}$ and $\mathrm{L}$ groups, respectively. Approximately $94.9 \%$ of the SNPs observed in the $\mathrm{H}$ group were also detected in the L group. Similarly, $94.8 \%$ of the SNPs in the $\mathrm{L}$ group were also detected in the $\mathrm{H}$ group.

For those SNPs detected across the off-target regions, the $\mathrm{M}$ group detected far more SNPs than the other two groups with 105,154 SNPs versus 78,745 and 70,766 per subject for $\mathrm{H}$ and L, respectively (Figure 3(a) and Supplementary Table 2). Almost all SNPs (97.9\%) detected in the $H$ and $L$ groups were also found in the M group, while only $73.5 \%$ and $66.2 \%$ of SNPs detected in the M group could be identified in the $\mathrm{H}$ and L groups, respectively (Figure 3(b)). Together, an average of $1,480,1,246$, and 23,397 SNPs across 92 subjects was uniquely detected in the $\mathrm{H}, \mathrm{L}$, and $\mathrm{M}$ group, respectively (Supplementary Table 2), where over $80 \%$ of the SNPs within each group were located in the off-target regions.
We then examined the SNP calling accuracy. The M group also showed better performance than the $\mathrm{H}$ and the $\mathrm{L}$ groups. For SNPs located in the target regions and uniquely observed within each group, the average $\mathrm{Ti} / \mathrm{Tv}$ ratios were $2.00 \pm 0.13$ (mean $\pm \mathrm{SD}), 1.39 \pm 0.21$, and $1.22 \pm 0.19$ for the $\mathrm{M}, \mathrm{H}$, and L groups, respectively (Figure 4(a)). For SNPs located in the off-target regions, the corresponding $\mathrm{Ti} / \mathrm{Tv}$ ratios were $2.09 \pm$ $0.03,1.46 \pm 0.09$, and $1.35 \pm 0.11$ for the $\mathrm{M}, \mathrm{H}$, and L groups, respectively (Figure $4(\mathrm{~b})$ ).

The Hete/Homo ratio was another parameter to evaluate the genotype quality with the expected value of 1.5 on a genomic scale $[26,27]$. Among the SNPs uniquely observed in the $\mathrm{M}$ group, the average Hete/Homo ratio was 1.51 and 1.52 in the on-target region and off-target regions, respectively (Figures 4(c) and 4(d)). However, the Hete/Homo ratios were much larger for SNPs uniquely observed in the $\mathrm{H}$ and $\mathrm{L}$ groups, being 10.45 and 22.43 across the on-target regions and 7.93 and 18.92 across the off-target regions. Among the SNPs uniquely discovered in the $\mathrm{M}$ group, $94.4 \%$ were included in the $1 \mathrm{KG}$ project. However, only $65.7 \%$ and $59.3 \%$ of the SNPs 


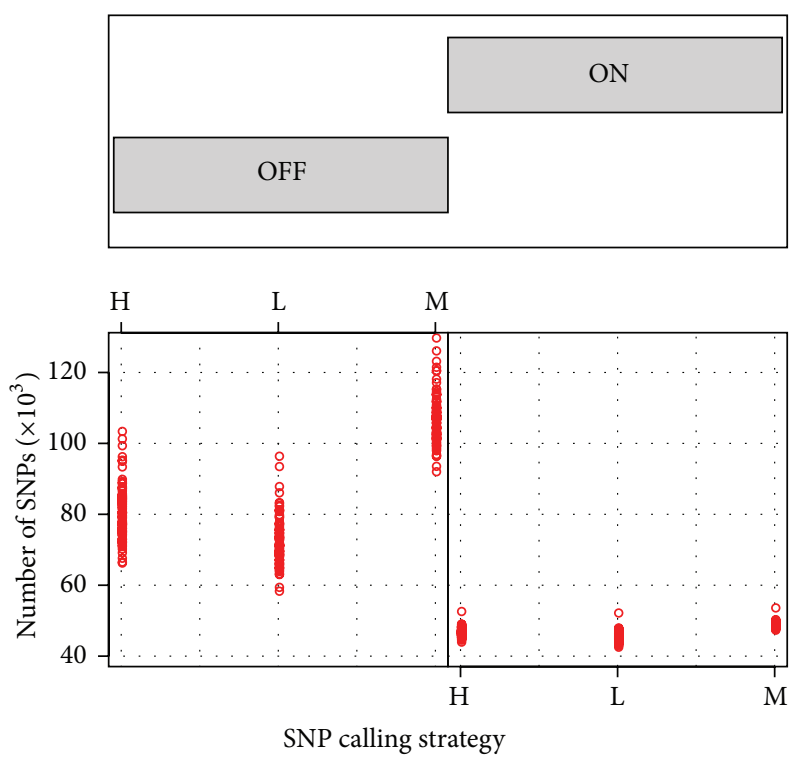

(a)

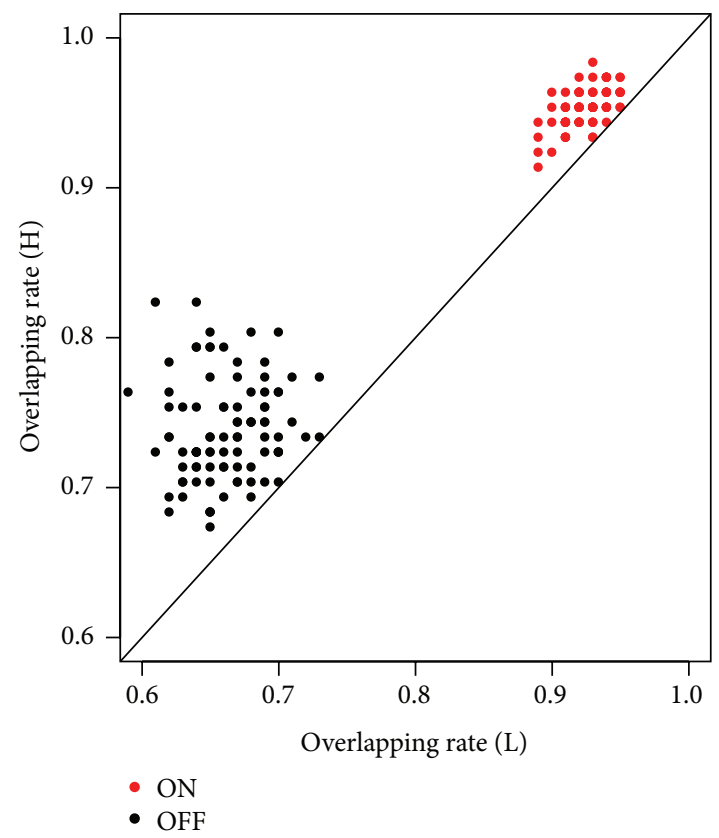

(b)

FIGURE 3: Comparison of SNPs identified across the on-target and off-target regions in three groups (M, L, and H). (a) Each red circle denotes the number of SNPs detected in the three groups within two regions (ON and OFF). (b) Overlapping rate of the SNPs detected in the H ( $y$ axis) and L ( $x$-axis) groups with the M group. Each dot denotes the overlapping rate of SNPs across the ON (red) and OFF (black) regions in each corresponding exome. ON and OFF represent the on-target and off-target regions, respectively; $\mathrm{M}, \mathrm{L}$, and $\mathrm{H}$ represent the merged, low depth, and high depth groups, respectively.

uniquely called in the $\mathrm{H}$ and $\mathrm{L}$ groups were overlapped with the SNPs in the $1 \mathrm{KG}$ project (Figures $4(\mathrm{e})$ and $4(\mathrm{f})$ ).

We finally compared the allele frequency of variants called by three approaches. Figure 5 shows the alternative allele (relative to the hg19 reference genome) frequency (AF) distribution among the SNPs observed in these three groups. For alternative alleles with $\mathrm{AF} \geq 0.1$, it showed that the allele frequency spectrum among three groups was overall identical, despite a slight difference in the range of 0.1-0.2. However, for low-frequency alleles ( $\mathrm{AF} \leq 0.05)$, we found the allele frequency is significantly lower (two-tailed Fisher's exact test, $P<0.001$ ) in the $\mathrm{M}$ group (Figure 5), almost up to 2 times lower than that in the $\mathrm{L}$ group. When the frequency of uniquely observed SNPs was considered, similar patterns were observed (Supplementary Figure 1), suggesting that merging duplicated exome-seq data within each subject would reduce false discovery rates for rare variants.

\section{Discussion}

In this study, we comprehensively investigated the variant calling based on three strategies to utilize the duplicated WES data from 92 subjects. From the aspects of the number of high quality variants, Hete/Homo ratio, Ti/Tv ratio, and overlapping rate with the $1 \mathrm{KG}$, our comparative analyses indicate that the $\mathrm{M}$ strategy (merging duplicates into one) is markedly superior to the other two approaches, especially for identifying variants located in the off-target regions.
The $\mathrm{Ti} / \mathrm{Tv}$ ratio is a critical metric for assessing the specificity of SNP calling [23]. The empirical Ti/Tv ratios are $\sim 2.0$ for genome-wide variants and $\sim 3.0-3.3$ for coding variants $[18,27]$. Typically, the $\mathrm{Ti} / \mathrm{Tv}$ ratio is lower in the newly discovered SNPs than that in known SNPs because of a combination of residual false positives, a relative deficit of transitions due to sequencing context bias, and an apparently higher transition ratio at lower frequency variation [23]. The $\mathrm{Ti} / \mathrm{Tv}$ ratio for SNPs uniquely called in the M group was much closer to expected value, suggesting the higher confidence of these SNPs than those uniquely called in $\mathrm{H} / \mathrm{L}$ groups. The Hete/Homo ratio, an average of $\sim 1.5$ on the genomic scale $[26,27]$, is another metric for assessing the SNP calling accuracy. The observed Hete/Homo ratio was $1.51 \pm 0.13$ ( \pm s.d.) for these SNPs uniquely observed in the M group. In addition, the overlapping rate between the SNPs uniquely detected in the $\mathrm{M}$ group and the $1 \mathrm{KG}$ project was much higher than that in the $\mathrm{L}$ and $\mathrm{H}$ groups. These results are consistent with Liu et al's report that multiple-sample calling by GATK pipelines increased the sensitivity of variants calling [29].

Although the aim of WES is to identify SNPs located in the coding regions, not all coding variants could be equally discovered. There are diverse reasons, including high or low GC contents in the target regions, uneven DNA capture, and sequencing depth [30-32]. Besides improving technical issues, the maximal utilization of WES data, particularly in the noncoding regions, is also of great interest. The enrichment of SNPs in the off-target regions might be related to the 


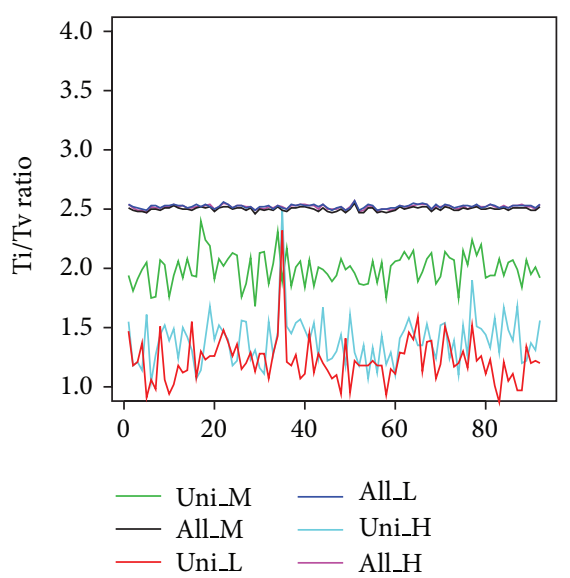

(a)

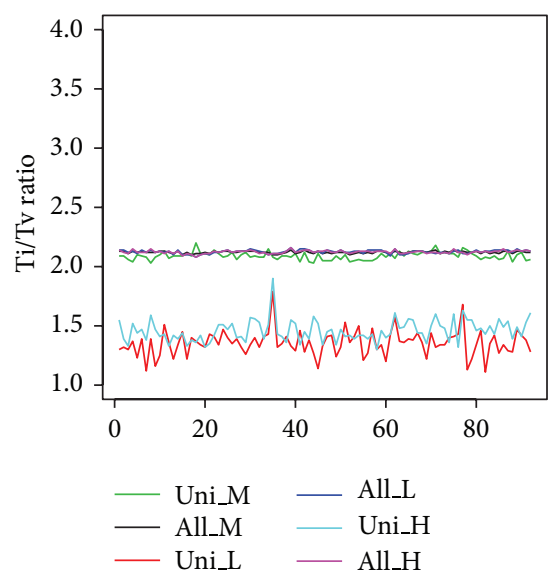

(b)

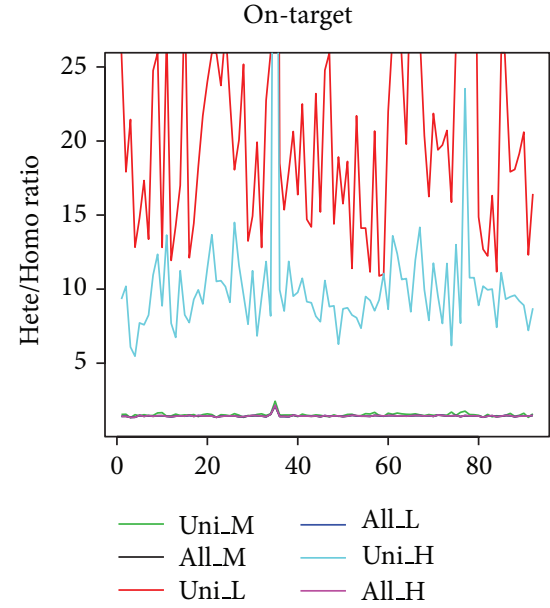

(c)

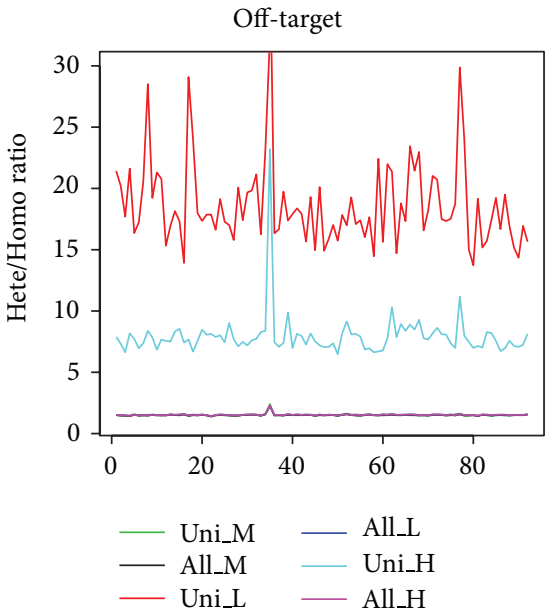

(d)

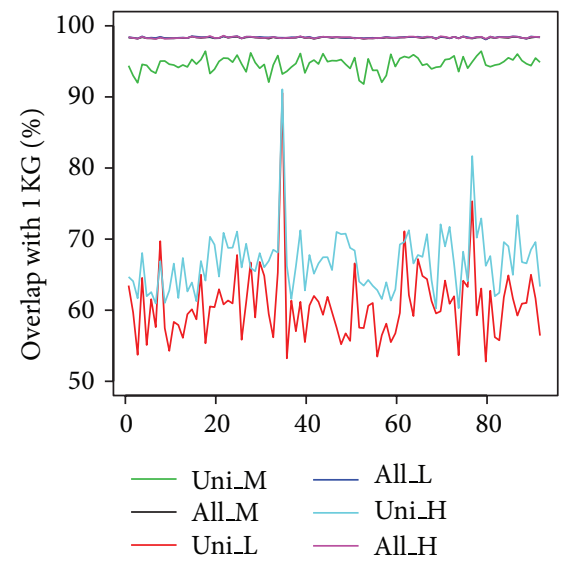

(e)

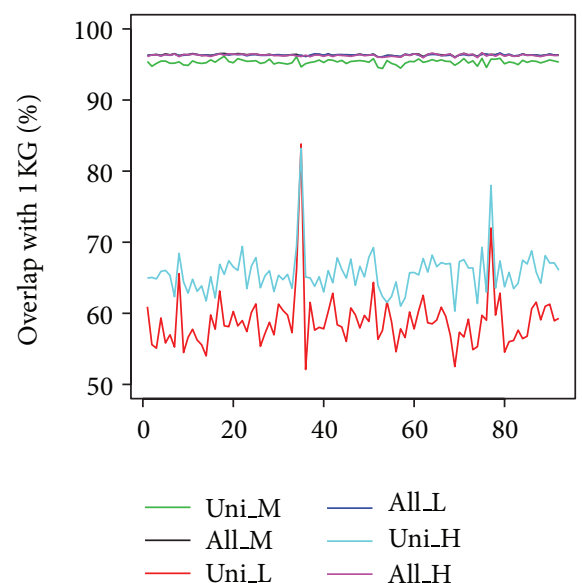

(f)

FIGURE 4: Comparison of the accuracy of SNPs detected by three strategies. The upper panel is plotted for SNPs across the on-target regions; the lower panel is for the off-target SNPs. From the left to right in each panel are the Ti/Tv ratio ((a) and (b)), Hete/Homo ratio ((c) and (d)), and overlapping rate with SNPs observed in the 1 KG project ((e) and (f)). Uni_M, Uni_L, and Uni_H represent the number of SNPs uniquely identified by the merged, low depth, and high depth strategies, respectively; All_M, All_L, and All_H denote the total number of SNPs identified by the merged, low depth, and high depth strategies, respectively. The $x$-axis represents each of 92 exome-seq samples.

capturing probe design and hybridization steps. Especially, increasing the sequencing depth (equivalent to the merged strategy) per sample may help to identify more off-target SNPs. SNPs in off-target regions are reported in multiple capture platforms, including Illumina, NimbleGen, and Agilent platforms $[17,18]$. However, SNPs located in off-target regions exhibit more variability in the number of variants and data quality. This suggests that more consideration should be taken into account when calling SNPs across the off-target regions. In the present study, we found that merging BAM files from duplicated sequencing data could greatly enhance the detection of high quality SNPs in off-target regions.

Merging two or more replicated sequencing data could improve SNP calling accuracy, as this strategy is to a large extent similar to the addition of sequencing depth. However, there is a prerequisite. It needs to assess the heterogeneity between replicates within each subject before merging them. Multiple measurements, including on-target region mapping rate, low quality read rate, the fraction of four bases, and genome coverage, could be used for evaluation. We also propose that the hierarchical clustering based on the RPKM statistic is a robust evaluation for quality control in the WES performance [25] as the RPKM statistic does for RNA-sequencing data $[33,34]$. In addition, due to the fact that replicated experiments are generated from the same sequencing library preparation, which shows the low batch problem, it is unclear whether merging replicated samples prepared from the different sequencing libraries also reaches this conclusion. As Leek et al. reported [35], large batch effects are associated with DNA preparation group and processing date. Therefore, if the two duplicates within each subject show remarkable disparity in the quality control, it is cautious to use merging strategy for further analysis.

In summary, our investigation indicates that merging the low heterogeneous duplicated WES data within each subject into a single sample and then conducting SNP calling 


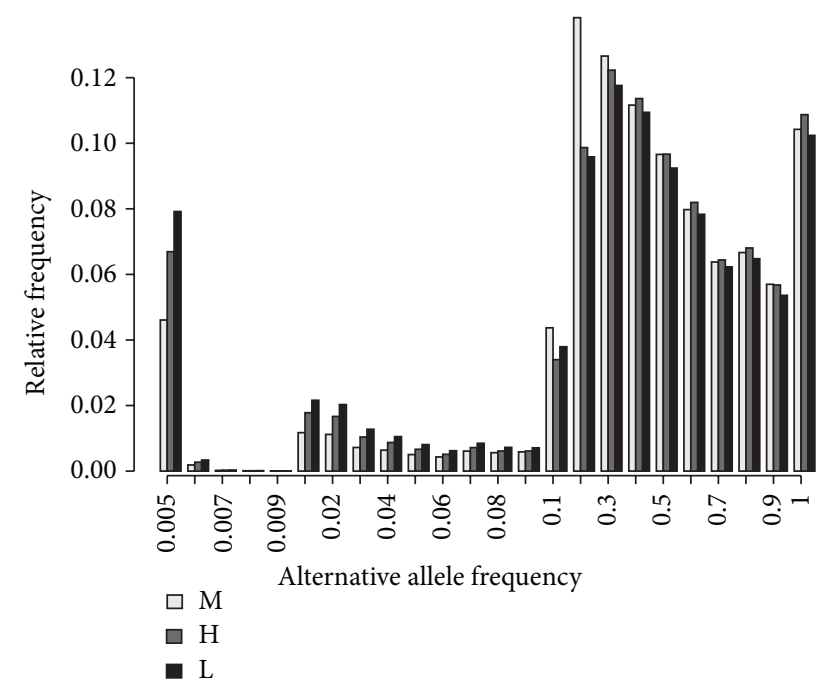

FIGURE 5: Alternative allele frequency distribution of all SNPs called in each of the three groups, $\mathrm{H}, \mathrm{L}$, and $\mathrm{M}$.

are a reasonable strategy to discover variants from the next generation sequencing technology.

$\begin{array}{ll}\text { Abbreviations } \\ \text { GATK: } & \text { Genome analysis toolkit } \\ \text { Hete/Homo: } & \text { Heterozygous-homozygous ratio } \\ \text { Ti/Tv: } & \text { Transition-transversion ratio } \\ \text { WES: } & \text { Whole-exome sequencing } \\ \text { SBCS: } & \text { Shanghai breast cancer study } \\ \text { H: } & \text { High depth } \\ \text { L: } & \text { Low depth } \\ \text { M: } & \text { Merged } \\ \text { RPKM: } & \text { Reads per kilobase and million mapped reads } \\ \text { 1 KG: } & \text { 1000 genomes. }\end{array}$

\section{Conflict of Interests}

The authors have no competing interests to declare.

\section{Authors' Contribution}

Jirong Long designed the study. Yanfeng Zhang performed computational analyses with the help of Bingshan $\mathrm{Li}$ and Chun Li. Yanfeng Zhang wrote the paper along with Jirong Long. Qiuyin Cai directed the lab operation. Wei Zheng directed the parent studies. All authors contributed to paper revision and approved the final paper.

\section{Acknowledgments}

The authors thank Regina Courtney and Jie Wu for their excellent laboratory supports. This research was supported in part by US National Institutes of Health grants R01CA158473 (to W. Zheng); R01CA137013 (to J. Long), and Ingram Professorship and Research Reward funds (to W. Zheng).
Sample preparation and genotyping assays at Vanderbilt were conducted at the Survey and Biospecimen Shared Resources and Vanderbilt Microarray Shared Resource, which are supported in part by the Vanderbilt-Ingram Cancer Center (P30CA068485).

\section{References}

[1] J. Shendure and H. Ji, "Next-generation DNA sequencing," Nature Biotechnology, vol. 26, no. 10, pp. 1135-1145, 2008.

[2] J. S. Parla, I. Iossifov, I. Grabill, M. S. Spector, M. Kramer, and W. R. McCombie, "A comparative analysis of exome capture," Genome biology, vol. 12, article R97, 2011.

[3] M. N. Bainbridge, M. Wang, D. L. Burgess et al., "Whole exome capture in solution with 3 Gbp of data," Genome Biology, vol. 11, no. 6, article R62, 2010.

[4] J. K. Teer and J. C. Mullikin, "Exome sequencing: the sweet spot before whole genomes," Human Molecular Genetics, vol. 19, no. 2, pp. R145-R151, 2010.

[5] W. Fu, T. D. O'Connor, G. Jun et al., "Analysis of 6,515 exomes reveals the recent origin of most human protein-coding variants," Nature, vol. 493, no. 7431, pp. 216-220, 2013.

[6] S. B. Ng, E. H. Turner, P. D. Robertson et al., "Targeted capture and massively parallel sequencing of 12 human exomes," Nature, vol. 461, no. 7261, pp. 272-276, 2009.

[7] X. Yi, Y. Liang, E. Huerta-Sanchez et al., "Sequencing of 50 human exomes reveals adaptation to high altitude," Science, vol. 329, no. 5987, pp. 75-78, 2010.

[8] J. A. Tennessen, A. W. Bigham, T. D. O’Connor et al., "Evolution and functional impact of rare coding variation from deep sequencing of human exomes," Science, vol. 337, no. 6090, pp. 64-69, 2012.

[9] T. W. Yu, M. H. Chahrour, M. E. Coulter et al., "Using wholeexome sequencing to identify inherited causes of autism," Neuron, vol. 77, no. 2, pp. 259-273, 2013.

[10] E. R. Thompson, M. A. Doyle, G. L. Ryland et al., "Exome sequencing identifies rare deleterious mutations in DNA repair genes FANCC and BLM as potential breast cancer susceptibility alleles," PLoS Genetics, vol. 8, no. 9, Article ID e1002894, 2012.

[11] S. J. Sanders, M. T. Murtha, A. R. Gupta et al., "De novo mutations revealed by whole-exome sequencing are strongly associated with autism," Nature, vol. 484, no. 7397, pp. 237-241, 2012.

[12] M. J. Bamshad, S. B. Ng, A. W. Bigham et al., "Exome sequencing as a tool for Mendelian disease gene discovery," Nature Reviews Genetics, vol. 12, no. 11, pp. 745-755, 2011.

[13] A. Kiezun, K. Garimella, R. Do et al., "Exome sequencing and the genetic basis of complex traits," Nature Genetics, vol. 44, no. 6, pp. 623-630, 2012.

[14] E. J. Vallender, "Expanding whole exome resequencing into non-human primates," Genome Biology, vol. 12, article R87, 2011.

[15] R. D. George, G. McVicker, R. Diederich et al., "Trans genomic capture and sequencing of primate exomes reveals new targets of positive selection," Genome Research, vol. 21, no. 10, pp. 16861694, 2011.

[16] J. E. McCormack, M. G. Harvey, B. C. Faircloth, N. G. Crawford, T. C. Glenn, and R. T. Brumfield, "A phylogeny of birds based on over 1,500 loci collected by target enrichment and highthroughput sequencing," PLOS ONE, vol. 8, no. 1, Article ID e54848, 2013. 
[17] M. J. Clark, R. Chen, H. Y. K. Lam et al., "Performance comparison of exome DNA sequencing technologies," Nature Biotechnology, vol. 29, no. 10, pp. 908-916, 2011.

[18] Y. Guo, J. Long, J. He et al., "Exome sequencing generates high quality data in non-target regions," BMC Genomics, vol. 13, article 194, 2012.

[19] Y. T. Gao, X. O. Shu, Q. Dai et al., "Association of menstrual and reproductive factors with breast cancer risk: results from the Shanghai breast cancer study," International Journal of Cancer, vol. 87 , no. 2, pp. 295-300, 2000.

[20] W. Zheng, J. Long, Y. Gao et al., "Genome-wide association study identifies a new breast cancer susceptibility locus at 6q25.1," Nature Genetics, vol. 41, no. 3, pp. 324-328, 2009.

[21] H. Li and R. Durbin, "Fast and accurate short read alignment with Burrows-Wheeler transform," Bioinformatics, vol. 25, no. 14, pp. 1754-1760, 2009.

[22] H. Li, B. Handsaker, A. Wysoker et al., "The sequence alignment/map format and SAMtools," Bioinformatics, vol. 25, no. 16, pp. 2078-2079, 2009.

[23] M. A. DePristo, E. Banks, R. Poplin et al., "A framework for variation discovery and genotyping using next-generation DNA sequencing data," Nature Genetics, vol. 43, no. 5, pp. 491-498, 2011.

[24] A. McKenna, M. Hanna, E. Banks et al., "The genome analysis toolkit: a MapReduce framework for analyzing next-generation DNA sequencing data," Genome Research, vol. 20, no. 9, pp. 1297-1303, 2010.

[25] P. Frommolt, A. T. Abdallah, J. Altmüller et al., "Assessing the enrichment performance in targeted resequencing experiments," Human Mutation, vol. 33, no. 4, pp. 635-641, 2012.

[26] S. C. Schuster, W. Miller, A. Ratan et al., "Complete Khoisan and Bantu genomes from southern Africa," Nature, vol. 463, no. 7283, pp. 943-947, 2010.

[27] K. J. McKernan, H. E. Peckham, G. L. Costa et al., "Sequence and structural variation in a human genome uncovered by short-read, massively parallel ligation sequencing using twobase encoding," Genome Research, vol. 19, no. 9, pp. 1527-1541, 2009.

[28] The 1000 Genomes Project Consortium, "An integrated map of genetic variation from 1,092 human genomes," Nature, vol. 491, no. 7422, pp. 56-65, 2012.

[29] X. Liu, S. Han, Z. Wang et al., "Variant callers for next-generation sequencing data: a comparison study," PLOS ONE, vol. 8, no. 9, Article ID e75619, 2013.

[30] A. Sirmaci, Y. J. K. Edwards, H. Akay, and M. Tekin, "Challenges in whole exome sequencing: an example from hereditary deafness," PLoS ONE, vol. 7, no. 2, Article ID e32000, 2012.

[31] M. N. Bainbridge, M. Wang, Y. Wu et al., "Targeted enrichment beyond the consensus coding DNA sequence exome reveals exons with higher variant densities," Genome Biology, vol. 12, no. 7, article R68, 2011.

[32] J. Freudenberg, P. K. Gregersen, and Y. Freudenberg-Hua, "A simple method for analyzing exome sequencing data shows distinct levels of nonsynonymous variation for human immune and nervous system genes," PLoS ONE, vol. 7, no. 6, Article ID e38087, 2012.

[33] A. Roberts, C. Trapnell, J. Donaghey, J. L. Rinn, and L. Pachter, "Improving RNA-Seq expression estimates by correcting for fragment bias," Genome Biology, vol. 12, no. 3, article R22, 2011.

[34] A. Mortazavi, B. A. Williams, K. McCue, L. Schaeffer, and B. Wold, "Mapping and quantifying mammalian transcriptomes by RNA-Seq," Nature Methods, vol. 5, no. 7, pp. 621-628, 2008.
[35] J. T. Leek, R. B. Scharpf, H. C. Bravo et al., “Tackling the widespread and critical impact of batch effects in high-throughput data," Nature Reviews Genetics, vol. 11, no. 10, pp. 733-739, 2010. 

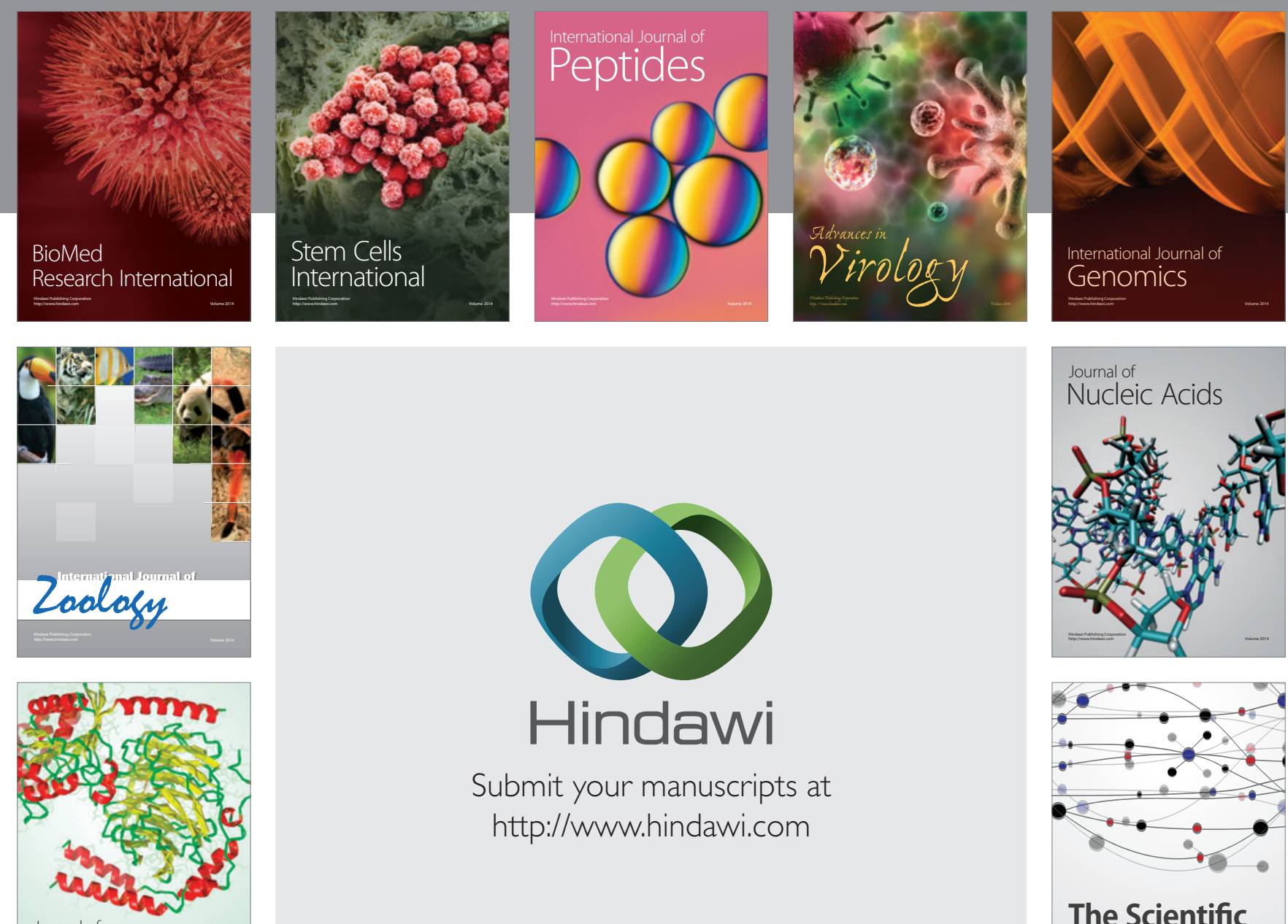

Submit your manuscripts at

http://www.hindawi.com

Journal of
Signal Transduction
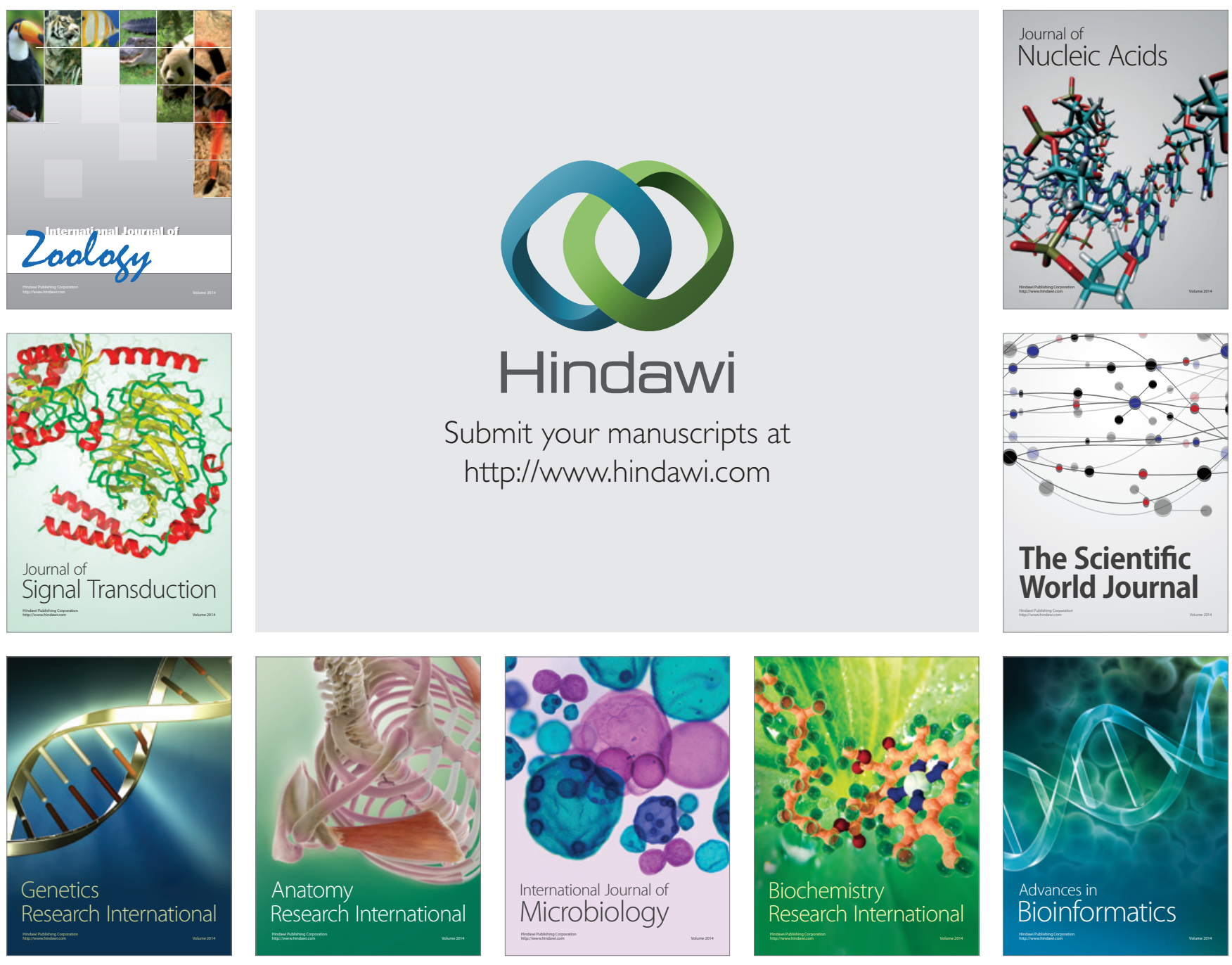

The Scientific World Journal
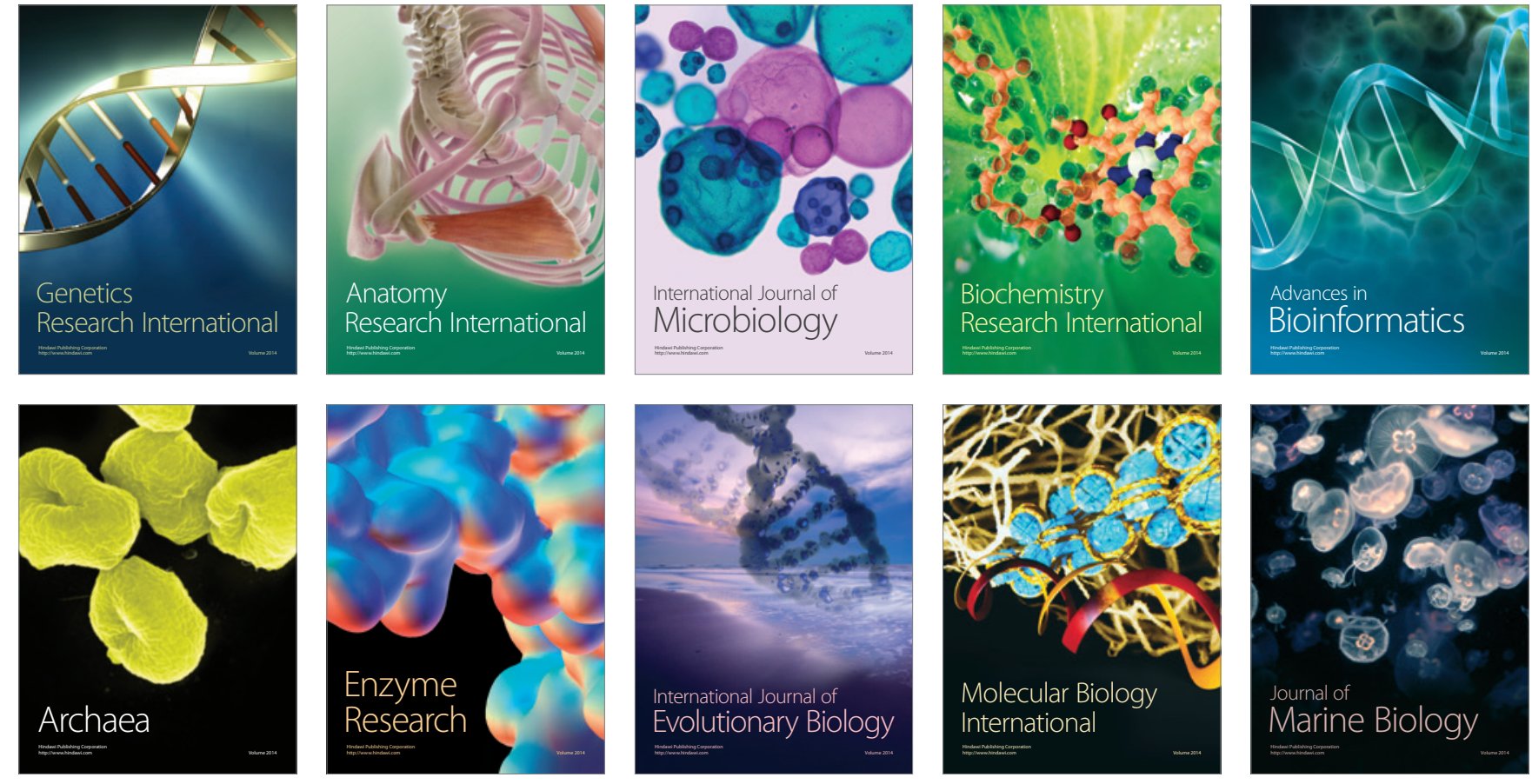